\title{
Turismo chino en itinerarios culturales: El Camino de Santiago y la Ruta Antigua de Té y Caballos
}

\author{
Jinjing Shen* Joaquín Beltrán Antolín**
}

Universitat Autónoma de Barcelona (España)

\begin{abstract}
Resumen: Este trabajo es un estudio comparativo de los turistas chinos que realizan el Camino de Santiago en España y la Ruta Antigua de Té y Caballos en China. Se parte de un breve estado de la cuestión sobre los itinerarios culturales como parte del turismo cultural, seguido de la evolución reciente del turismo chino interno e internacional para, a continuación, analizar la construcción/creación del Camino de Santiago y la Ruta Antigua de Té y Caballos como destinos turísticos. Finalmente se compara el perfil de los turistas en ambas rutas incluyendo sus motivaciones, experiencias y modo de acceso a la información para realizar este tipo de viaje que se ha convertido en un símbolo de distinción y de clase para una minoría de la población y se encuadran en la nueva tendencia de turismo independiente y experiencial que se está desarrollando en China.
\end{abstract}

Palabras Clave: Turismo chino; Itinerario cultural; Camino de Santiago; Ruta Antigua de Té y Caballos; Turismo independiente; Turismo de experiencias; China.

\section{Chinese Tourism in Cultural Routes: The Camino de Santiago and the Ancient Tea Horse Road}

Abstract: This paper is a comparative study of Chinese tourists in the Camino de Santiago in Spain and the Ancient Tea Horse Road in China. First, we introduce a brief state of the arts on cultural routes as part of cultural tourism, and on the recent evolution of inbound and outbound Chinese tourism. Second, we analyze the construction / creation of the Camino de Santiago and the Ancient Tea Horse Road as tourist destinations. Finally, we compare the profile of the tourists on both routes, including their motivations, experiences and the way to access to the necessary information to travel. The cultural routes have become a symbol of distinction and class for a minority of the population and are framed in the new trend of independent and experiential tourism that is developing in China.

Keywords: Chinese tourism; Cultural routes; Camino de Santiago; Ancient Tea Horse Road; Independent tourism; Experiential tourism; China.

\section{Introducción}

Los itinerarios culturales fueron institucionalizados y legitimados por primera vez por el Consejo de Europa en 1987 al aprobar y declarar al Camino de Santiago como el primer Itinerario Cultural Europeo. Posteriormente la UNESCO lo incluyó en la lista del Patrimonio Mundial en 1993. Los itinerarios culturales pasaron a ser identificados con una categoría propia a mediados de la década de 1990 y desde entonces su certificación, solicitada por cada vez más rutas en todo el mundo, ha tenido consecuencias en el desarrollo del sector turístico a escala local y global al convertirse en un reclamo para su visita. En definitiva, los itinerarios/rutas culturales se han consolidado como un tipo específico de turismo cultural y de patrimonio que a menudo también incluye atractivos relacionados con la naturaleza.

El objetivo de este trabajo es el análisis comparativo de dos itinerarios/rutas culturales en relación con el turismo chino: el Camino de Santiago, en España, y la Ruta Antigua de Té y Caballos, en la República Popular China. Ambos caminos sagrados-comerciales se convirtieron a partir de la década

\footnotetext{
* Universitat Autònoma de Barcelona (España); E-mail: afeijj223797@hotmail.com

* Universitat Autònoma de Barcelona (España); E-mail: JoaquinBeltran@uab.cat
} 
de 1990 en focos de atracción para el turismo cultural con un alcance local y global. El nexo para la comparación será las características del turismo y de los turistas chinos que los realizan. China ha entrado en la escena mundial como origen y destino turístico pasando a ocupar posiciones destacadas en los flujos turísticos globales, al mismo tiempo que desarrolla de un modo acelerado su turismo interno.

El turismo chino se ha convertido en un fenómeno de consumo de masas conforme los ingresos de la población del país se han elevado de forma generalizada. En este contexto las ofertas y destinos turísticos se han diversificado y segmentado para satisfacer a la creciente demanda con necesidades y expectativas diferenciadas. Los itinerarios culturales basados en caminos históricos constituyen todavía una novedad para el turismo chino interno e internacional, por lo que el volumen de las personas que los realizan todavía es muy pequeño. Estos viajes se han convertido en un símbolo de distinción y de clase para una minoría de la población y se encuadran en la nueva tendencia de turismo de carácter más o menos independiente y experiencial que se está desarrollando en China.

Para llevar a cabo la comparación se partirá de un breve estado de la cuestión sobre los itinerarios culturales como parte del turismo cultural, seguido de la evolución reciente del turismo chino interno e internacional para, a continuación analizar la construcción/creación del Camino de Santiago y de la Ruta Antigua de Té y Caballos como destinos turísticos, para finalmente comparar las características de los turistas chinos que las realizan, incluyendo sus fuentes de información previas al viaje, el perfil de los turistas y su comportamiento.

\section{Itinerarios/rutas culturales y turismo}

El turismo cultural es una actividad que incluye experimentar la cultura local del lugar que se visita, acercarse y conocer las tradiciones y el estilo de vida, participar en actividades relacionadas con el arte y las visitas a museos, monumentos y sitios del patrimonio. La definición adoptada por la Asamblea General de la UNWTO (Chengdu, 11-16 de septiembre de 2017) fue:

"Cultural tourism is a type of tourism activity in which the visitor's essential motivation is to learn, discover, experience and consume the tangible and intangible cultural attractions/products in a tourism destination. These attractions/products relate to a set of distinctive material, intellectual, spiritual and emotional features of a society that encompasses arts and architecture, historical and cultural heritage, culinary heritage, literature, music, creative industries and the living cultures with their lifestyles, value systems, beliefs and traditions" (World Tourism Organization, 2018: 11).

El turismo cultural también es "aquel viaje turístico motivado por conocer, comprender y disfrutar el conjunto de rasgos y elementos distintivos, espirituales y materiales, intelectuales y afectivos que caracterizan a una sociedad o grupo social de un destino específico" (Cestur-Sectur, 2002: 5, citado en Fernández Salinas, 2013). En definitiva, es el movimiento de personas a lugares de interés alejados de su lugar de residencia habitual, con la intención de acceder a conocimientos y tener experiencias nuevas para satisfacer sus necesidades culturales (Richards, 2001). El turismo cultural busca explotar el valor de los productos culturales al máximo y, al mismo tiempo, define de nuevo las experiencias de los viajeros y la influencia de la cultura en la industria del turismo. Por eso, el turismo moderno fusiona los turistas, la cultura y el viaje (Craik, 1997).

Por su parte, los itinerarios o rutas turísticas son trayectos o caminos en los que se especifican los lugares de paso y donde, además, se proponen una serie de actividades y servicios (Gómez Prieto y González-Quijano, 1991). La diferencia entre itinerarios/rutas con respecto a otro tipo de movilidad turística es la capacidad de recorrer y al mismo tiempo ver, conocer y sentir (Torres Bernier, 2006). Los itinerarios a lo largo de la historia "han facilitado la expansión de las culturas y han propiciado los mestizajes culturales" (Fernández Salinas, 2013).

Después de que UNESCO incluyera en la lista del Patrimonio Mundial en 1993 al Camino de Santiago -previamente declarado como el primer Itinerario Cultural Europeo por el Consejo de Europa en 1987-, la Comisión Ejecutiva del Patrimonio Mundial (UNESCO) organizó en Madrid en 1994 un encuentro sobre "Los Itinerarios como Patrimonio Cultural" en cuya conclusión se propuso una definición de las rutas culturales como rutas transnacionales (Capel, 2005) que a su vez fue la base para la Asamblea General del ICOMOS (Internacional Council on Monuments and Sites) de 2005 sobre este tema. Casi al mismo tiempo se crearon el Instituto Europeo de Itinerarios Culturales de la Unión Europea (1997) y el Comité Científico Internacional de Itinerarios Culturales (CIIC) de ICOMOS (1998). La Carta 
internacional sobre los itinerarios culturales propuesta por el CIIC en 2005 introducía esta nueva categoría en el marco del Patrimonio Mundial de la UNESCO, siendo ratificada finalmente en 2008 (Capel, 2005; Hernández Ramírez, 2011; Morère Molinero, 2012; Fernández Salinas, 2013).

Las rutas son parte de la actual consideración del patrimonio, donde el territorio es un componente patrimonial más extenso que incluye programas, políticas culturales y turísticas (Fernández-Baca, 2014). Las rutas ya no hacen solo mención al circuito, también son el objeto del recorrido (Calabuig Tomás y Ministral Masgrau, 1998), son el destino en sí mismas.

Los itinerarios y rutas turísticos-culturales se han convertido en la actualidad en uno de los recursos turísticos con mayor crecimiento. Diferentes instituciones tanto de ámbito local como nacional e internacional han apostado firmemente por su creación y diseño evidenciando y valorizando sus recursos (ICOMOS, 2008). El objetivo es promover el patrimonio en dichos territorios, así como fortalecer y/o crear sentimientos de identidad (López-Guzmán, Lara de Vicente y Merinero-Rodríguez, 2006), a lo que se añade también el fomento del desarrollo sostenible a partir de la comercialización de los recursos del territorio (Hernández Ramírez, 2011). El diseño de las rutas para su reconocimiento y explotación es complejo, y en muchas ocasiones las propuestas sometidas a aprobación se limitan a un inventario de elementos que comparten un tema común, aunque realmente sean inconexos.

En definitiva, como Capel (2005) señala:

"Declarar como Patrimonio de la Humanidad a rutas o itinerarios culturales de carácter transnacional pone el énfasis no solo en los valores culturales o históricos de determinados espacios y paisajes, sino también en 'los movimientos de población, el encuentro, el diálogo, el cambio y la interfecundación de las culturas en el espacio y en el tiempo', a la vez que se insiste en la importancia que tienen esos itinerarios 'para la comprensión mutua, la lectura plural de la historia y la cultura de la paz"'.

El Camino de Santiago fue el primer itinerario cultural reconocido como Patrimonio de la Humanidad (1993), posteriormente se aprobaron otros como la Ruta de la Seda: red de rutas del corredor Chang'an-Tian Shan (2014) de 5.000 kilómetros que abarca lugares de China, Kazajistán y Kirguistán después de un intenso y largo trabajo investigación que comenzó en 1988 (UNESCO, 2002). La Ruta Antigua de Té y Caballos, también localizada en China y con conexión a otros países ya ha iniciado el proceso para solicitar su inclusión en el listado de la UNESCO.

\section{Turismo chino interno e internacional}

El turismo en China es un fenómeno relativamente reciente. En la década de 1990, tras el cambio de agenda económica de desarrollo del país iniciado en 1979, se encontraba en una fase inicial para progresivamente durante el siglo XXI pasar a otra de rápido crecimiento que ya lo ha convertido en un fenómeno de consumo de masas. Los avances del sector turístico chino se deben al aumento de los ingresos y del tiempo de ocio de la población y también al desarrollo de la economía del país (Ning, 2000; Zhang, 2002). De acuerdo a los datos oficiales publicados por The Yearbook of China Tourism Statistics (2001-2016) el volumen del turismo chino interno pasó de 744 millones personas en el año 2000 a 4.000 millones en 2015, multiplicándose por 5,4 veces en tan solo quince años. Este crecimiento se explica fundamentalmente por la mayor disponibilidad económica de la población del país: el PIB per cápita durante el mismo periodo pasó de 959 US\$ a 8.167 US\$, es decir se multiplicó por 8,5 veces. En realidad los datos anteriores no son reales pues contabilizan todos los movimientos de la población independientemente de que el motivo sea específicamente turístico. En cualquier caso tienen el valor de indicador de la movilidad.

La Ruta Antigua de Té y Caballos, objeto de esta investigación, une fundamentalmente a las provincias de Yunnan y Sichuan con la Región Autónoma de Tibet, por lo que el volumen del turismo interno en estas zonas incluye a los turistas que la realizan. De acuerdo a los datos de 2015 Sichuan recibía el 14,75\% del total del turismo chino; Yunnan el 8,08\% y Tibet el 0,5\%, aunque este porcentaje por si solo representa a 20,2 millones de viajeros. De hecho Tibet es la región que porcentualmente más ha crecido de las tres en la recepción turística durante los últimos años. En su conjunto, Yunnan, Sichuan y Tibet son el destino para el 23\% del turismo interno chino, y quienes realizan la Ruta tan solo representan una parte muy pequeña de ese porcentaje. Esta zona localizada en el oeste y suroeste de China se caracteriza por una gran diversidad étnica y cultural cuyo "exotismo" es explotado por el sector turístico. Además, especialmente el territorio que atraviesa la Ruta, coincide con zonas del 
país consideradas pobres donde el Estado promueve el desarrollo turístico como alternativa para el crecimiento económico.

Por su parte, el turismo chino internacional comenzó con una etapa inicial de prueba (1983-1996) en la cual se permitía salir del país a visitar a parientes a unos destinos concretos -Hong Kong, Macao, Thailandia, Malaysia, Singapur, Filipinas- y a partir de 1987 también se aprobaron las visitas cortas en las zonas de frontera -Corea del Norte, Mongolia, Rusia, Vietnam, Laos, Mynamar-. Durante la segunda fase (1997-2001) se abrió el turismo al exterior a partir de las "Provisiones sobre la Gestión del Viaje al Extranjero de los Ciudadanos Chinos" (1997) (Andreu, Claver y Quer, 2013). Se creó el Estatus de Destino Aprobado (EDA) que progresivamente incluyó a más países. En este periodo se aprobó el viaje turístico a los siguientes países: Corea del Sur, Australia, Nueva Zelanda, Japón, Vietnam, Camboya, Myanmar, Brunei, Malta, Indonesia, Turquía y Nepal. La tercera fase (2002-actualidad) es la de la consolidación del turismo hacia el extranjero, al pasar de 18 países con el EDA en 2001 a 150 a finales de 2014. En 2004 se añadieron 61 países a esta lista, entre ellos España, Francia, Italia, Portugal, etc. (World Tourism Organization, 2017).

"Looking back at the development of China's outbound travel market, it took eight years (1993-2000) for the annual number of outbound tourists to reach 10 million, only three more years (2001-2003) to reach 20 million, seven years (2004-2010) to climb to 50 million and four more years (2011-2014) to break 100 million record" (World Tourism Organization, 2017: 25)

Gráfico 1: Turismo chino internacional (en millones). Años 1993-2014

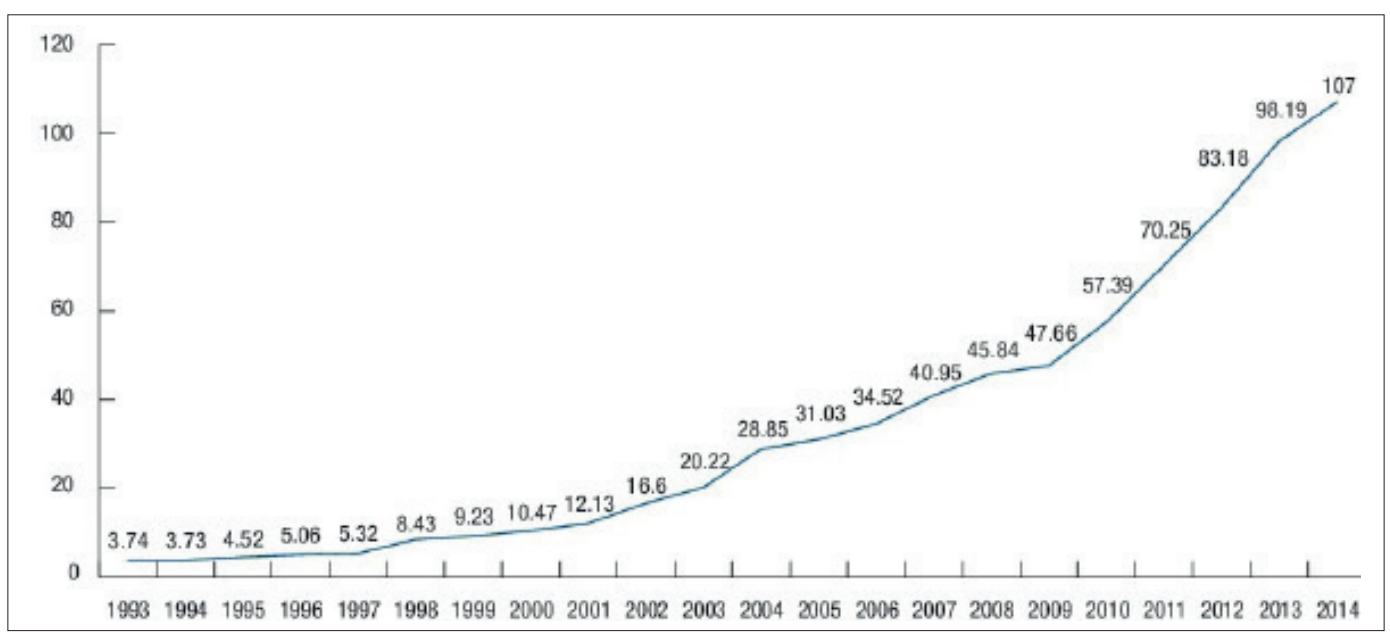

Fuente: World Tourism Organization, 2017, p. 25 (a partir de The Yearbook of China Tourism Statistics, 1994-2015)

El turismo chino internacional en 2015 fue de 120 millones de personas y una de sus principales tendencias es que en el $80 \%$ de los casos se lleva a cabo de forma independiente, es decir no sujeto exclusivamente a las ofertas de los operadores y agencias turísticas, ni a los viajes en grupo organizados que caracterizaban a las primeras fases del turismo en China cuando los viajeros contaban con una escasa experiencia turística, de información sobre los destinos, falta de dominio de idiomas extranjeros y dificultades para la preparación del viaje, por ejemplo, para hacer reservas (Lui et al., 2011). Además, hasta bien entrado el siglo XXI, la capacidad de consumo de servicios turísticos para la mayor parte de la población era relativamente baja, lo que facilitaba que el turismo de viaje intensivo en grupo para visitar lugares -sightseeing- fuera el más popular por su precio competitivo que resultaba atractivo en el sentido de gastar lo menos posible para disfrutar y visitar más destinos turísticos: por ejemplo, las ofertas de "10 países en 12 días" y el "tour sin comisiones" (Arlt y Aceves Leyva, 2011). Además también estaban especialmente interesados en realizar compras durante el viaje (Hotels.com, 2013). 
Actualmente el turismo chino interno e internacional mantiene un rápido y estable crecimiento anual con previsiones de continuidad al mismo ritmo a largo plazo y se ha convertido en un sector importante para el desarrollo de la economía nacional al impulsar industrias relacionadas y complementarias (Zhang, 2002; Hernández, 2018). El aumento del turismo internacional chino va acompañado por el creciente interés y valoración de experiencias y visitas profundas a destinos turísticos, además de la libertad en el proceso del viaje. El viaje con ritmo lento, tanto nacional como internacional, ya es una tendencia igual que el turismo de forma independiente (自助游, zizhuyou) acompañado de amigos o familiares y en coche propio (Lai, 2015).

España, de acuerdo a Turespaña (Instituto de Turismo de España), recibió a 513.725 turistas chinos en 2017 frente a 399.741 en 2015, año en que se multiplicaba por más de diez los 33.426 que llegaron en 2005. Otras fuentes, como ATEC (Asociación de Turismo España-China) afirman que en 2017 llegaron 718.000 turistas chinos a España (Hernández, 2018). La discrepancia de los datos se debe a que registran distintos movimientos: el primero contabiliza sólo a los que entran directamente procedentes de China y el segundo a los que también proceden de otros países europeos donde fueron previamente como primer destino -paquete turístico de "un viaje, varios países".

Según un estudio acerca de la imagen de España en China (Noya et al, 2007) y también de acuerdo a un trabajo de la consultora Taylor Nelson Sofres China (TNS) del año 2008 (Laso y Justo, 2009), España era el quinto destino turístico preferido de Europa para los turistas chinos, detrás de Francia, Reino Unido, Alemania e Italia y por delante de Holanda, Suiza y Austria, y ocupaba la treceava posición en el ranking de los 25 destinos internacionales turísticos más populares para ellos.

“...la imagen turística de España en China se asocia mayoritariamente con los paisajes y la naturaleza $(89 \%)$, la cultura y los museos (71\%) y con el sol y la playa (67\%). Un $15 \%$ de los encuestados afirma querer venir de turismo a España, por detrás de Francia (32\%) o EEUU (21\%) pero por delante de Italia (13\%) o Reino Unido (12\%). Un $76 \%$ de los encuestados lo considera atractivo como destino turístico, aunque la seguridad y el precio son percibidos como desincentivos" (Tomás Gaimundiz, 2011:33).

"[en 2014] La estadística sugiere que España ha sido un destino menos atractivo para los turistas chinos que otros países europeos, como Francia, Suiza, Italia y Alemania. Sin embargo, el efecto del turismo chino en la economía de España es significativo, ya que los turistas chinos tienen un gasto medio de $2.040 €$, dos veces más que el gasto de los alemanes en España" (Lojo, 2016: 513).

En el año 2014, los principales destinos turísticos de España fueron Cataluña (61,3\%), Comunidad de Madrid (22,8\%) y Andalucía (8,7\%) (Vivential Value, 2016). En cuanto al perfil del turista chino en España en 2010 para un 68,36\% fue su primer viaje al extranjero; viajan acompañados de familiares/ cónyuges (46,98\%), amigos (23,81\%), compañeros de trabajo/estudios (13,66\%) o de forma individual (6,95\%). De acuerdo al presidente de la Asociación de Turismo España China (ATEC) el turista chino en España:

"busca experiencias y gratificación intelectual (gastronomía, cultura, arte, espiritualidad, naturaleza, compras), se siente muy atraído por nuestro país (para ellos es un destino exótico), es cosmopolita y digital, busca interactuar con la comunidad local, estacionalidad diferente (febrero, principalmente)" (Prnoticias, 2018).

\section{El Camino de Santiago y la Ruta Antigua de Té y Caballos}

El Camino de Santiago es una ruta de peregrinación religiosa entre España y otros países europeos con destino final en Santiago de Compostela donde se veneran las reliquias del apóstol Santiago el Mayor (Sánchez, 2005). El descubrimiento de los restos de Santiago apóstol a principios del siglo IX marcó el inicio de las peregrinaciones a Compostela, estableciéndose el Camino de Santiago (Cànoves Valiente y Blanco Romero, 2011). Los peregrinos siguen muchas rutas de peregrinación, pero la más popular es la Vía Regia y su última parte, el Camino Francés. El Camino se realiza a pie, pero actualmente también algunos viajeros lo hacen en bicicleta o a caballo (Agís Villaverde, 2008).

La Ruta de Té y Caballos, por su parte, es una ruta comercial china que comenzó durante las dinastías Tang y Song (960-1279) para abastecer a la población tibetana de té que no se cultivaba en su región por las condiciones geográficas de elevada altura. El consumo de té ayuda a asimilar las grasas y a controlar las calorías de la dieta cotidiana de los tibetanos para quienes constituye una bebida de su 
dieta cotidiana. Por su parte las regiones del interior de China necesitaban numerosos mulos y caballos para el transporte civil y militar, con una demanda que superaba a la oferta. Y es precisamente en las regiones altas de Tibet donde la población ganadera local criaba caballos de calidad, frente a las provincias vecinas de Sichuan y Yunnan donde se producía té, sal y hierbas medicinales. Por eso se desarrolló el mercado complementario de intercambio entre té y caballos llegando a ser muy popular y activo (Chen, 2004, Cubeiro, 2015). Desde la antigüedad la Ruta de Té y Caballos tenía tres ramas principales: 1) desde la provincia de Yunnan a Tibet; 2) desde la provincia de Sichuan a Tibet; y 3) desde la provincia de Qinghai a Tibet (Luo, 2003). La ruta además se expandía para incluir a países vecinos que también intercambiaban todo tipo de productos con el imperio chino (Cubeiro, 2015).

El Camino Francés de Santiago y las rutas francesas del Camino fueron declarados Patrimonio de la Humanidad por la UNESCO en 1993 y 1998, respectivamente. En el Año Santo Jubilar Compostelano de $1993,{ }^{1}$ el gobierno autónomo de Galicia decidió convertir el Camino en un recurso turístico, abriéndolo a personas con un perfil diferente al tradicional peregrino religioso, mejorando sus infraestructuras y recuperando albergues y restaurantes. Una parte importante del desarrollo y la difusión del Camino de Santiago, tanto en España como en el resto del mundo, se debe a las Asociaciones de Amigos del Camino de Santiago (Zhang, 2015). En 1950 se fundó la primera en París. El I Encuentro Mundial de Asociaciones de Amigos del Camino se celebró en 2015 en Santiago de Compostela, asistieron 28 países y 157 asociaciones. En ese momento había registradas 350 asociaciones en todo el mundo -40\% de ellas en España-. ${ }^{2}$ Y en 2017 se fundó la Asociación de Amigos de China (Estella, 2017). La concepción originaria del Camino como una modalidad de turismo religioso ha evolucionado hacia un significado cultural más amplio, aportando así una nueva dimensión. Al mismo tiempo, el Camino se ha convertido en un factor de desarrollo local de los espacios rurales por donde pasa (Andrade Suárez, Leira Landeira y Caramés Valo, 2010).

Los turistas chinos que han realizado el Camino han pasado de 15 el año 2004 a 1.045 en 2017 (Informe Estadístico de la Oficina del Peregrino). Otros países de Asia Oriental superan a China en este viaje. El promedio de la población de Corea del Sur y de Japón cuenta con mayor experiencia turística y más capacidad consumo y sus intercambios con España han sido más intensos. Japoneses y coreanos conocen mejor la cultura, tradiciones, arte e historia de Europa y de España y la publicación de libros y producción de películas y documentales sobre el Camino han sido determinantes para darlo a conocer en sus países y suscitar el interés para realizarlo.

Tabla 2: Turistas coreanos, japoneses y chinos en el Camino de Santiago. Años 2004-2017

\begin{tabular}{|c|c|c|c|c|}
\hline & Total & Corea Sur & Japón & China \\
\hline 2004 & 179.944 & - & 257 & 15 \\
\hline 2005 & 93.924 & 28 & 278 & 5 \\
\hline 2006 & 100.377 & 84 & 282 & 10 \\
\hline 2007 & 114.026 & 449 & 327 & 19 \\
\hline 2008 & 124.141 & 915 & 412 & 20 \\
\hline 2009 & 145.877 & 1.079 & 526 & 35 \\
\hline 2010 & 272.135 & 1.493 & 796 & 59 \\
\hline 2011 & 183.366 & 1.740 & 877 & 112 \\
\hline 2012 & 192.488 & 2.493 & 860 & 186 \\
\hline 2013 & 215.880 & 2.774 & 840 & 431 \\
\hline 2014 & 237.983 & 3.842 & 1.095 & 510 \\
\hline 2015 & 262.516 & 4.073 & 1.179 & 706 \\
\hline 2016 & 277.854 & 4.534 & 1.459 & 831 \\
\hline 2017 & 301.036 & 5.106 & 1.474 & 1.045 \\
\hline
\end{tabular}

Fuente: Elaboración propia a partir de Informe Estadístico. Oficina del Peregrino. Varios años 
El origen de la Ruta Antigua de Té y Caballos como destino turístico es más reciente. Un equipo de investigación de la Universidad de Yunnan realizó un viaje por la ruta en 1990 utilizando el equipamiento de las antiguas caravanas que lo transitaban. Su recorrido de 2.000 kilómetros pasó por Zhongdian, Changdu, Montaña Hengduan y Kangding para finalmente volver a Zhongdian con el objetivo de verificar y recuperar la Ruta de Té y Caballos. Después de su expedición de tres meses los investigadores denominaron a este camino y red de intercambio comercial "Ruta Antigua de Té y Caballos" (Mu, 2001; Chen, 2005). En chino se denomina Chama gudao (茶马 古 道, Ruta Antigua de Té y Caballos), literalmente cha es té, ma es caballo, gu antiguo y dao camino o ruta. El camino era conocido tradicionalmente como Chamadao (茶马 道), pero ellos propusieron añadir la palabra "antiguo" (古 gu) al nombre original para recordar la larga historia de esta ruta (Sigley, 2010), lo cual además le añade un importante atractivo y es un reclamo para el turismo.

El cambio de nombre realizado a finales de la década de 1990 fue una estrategia premeditada por parte de los investigadores y del Estado para recuperar y desarrollar una ruta comercial histórica y convertirla en una ruta turística. La referencia a "lo antiguo", en el contexto cultural y social chino, se asocia a algo que resulta atractivo y que motiva a conocerlo y visitarlo. En el mismo sentido, una de las ciudades clave por la que transcurre la ruta, Zhongdian, en la provincia de Yunnan cerca de la frontera con Tibet, el año 2002 cambió oficialmente su nombre para rebautizarse como "Shangri-La" (香 格里 拉, Xianggelila). La ciudad mítica de Shangri-La posee toda una serie de connotaciones llenas de exotismo a partir de la novela Horizontes perdidos (1933) de James Hilton. El Estado chino decidió que fuera el nombre de una ciudad real con el objetivo fundamental del desarrollo turístico (Kolås, 2004; Bovair, 2008; Llamas y Belk, 2011).

"In the far reaches of Northwest Yunnan, the making of Xianggelila is a story of how local entrepreneurs, some Tibetan, many not, imaginatively linked themselves to this grandest of European colonial fantasies, to the British novel and popular U.S. film (translated into Chinese and widely distributed). This recuperated colonial past was then grafted onto a developmental model for the ethnic borderlands that would take mass tourism as the key link. Te aim was nothing less than to transform a little-known town in the northwest corner of Yunnan into a major tourist destination" (Litzinger, 2014, p. 281).

En 2007 se produjo otro cambio de nombre en la zona: la Municipalidad a nivel de prefectura Simao (思茅市) recuperó su antigua denominación Pu'er (普洱市) que es como se conoce a la variedad de té regional. Pu'er es también uno de los puntos de partida y ciudades-mercado clave de la Ruta Antigua de Té y Caballos (Zhang, 2014; Sigley, 2015).

"With the growth in domestic interest both in the Chama gudao and in Pu'er tea (which went from relative obscurity in the 1990s to one of the hottest trends in tea consumption and investment in recent years) tourism authorities (government and business developers) have been keen to 'develop' tourism sites and activities that link directly to the Ancient Tea Horse Road” (Sigley, 2010: 541).

Desde el año 1950 hasta el año 1976, las autopistas nacionales números 318, 317 y 214 entre Sichuan-Tíbet y Yunnan-Tibet se construyeron gradualmente. En torno al cambio de siglo comenzaron a elaborarse medidas para promocionar la recientemente designada "Ruta Antigua de Té y Caballos". Diferentes gobiernos locales utilizaron este nuevo nombre con el objetivo del desarrollo turístico de sus zonas, por ejemplo en 2001 el gobierno de Changdu de Tíbet encargó al Departamento de la Geografía de la Academia China de Ciencias Sociales planificar el turismo de la "Ruta Antigua de Té y Caballos". El modelo de "Transporte + Turismo" fue el principio adoptado ${ }^{3}$ (Zhang y Ma, 2006). En 2009, la Administración Nacional China de Turismo le concedió a la Ruta Antigua de Té y Caballos la categoría Premium o de primer nivel a escala nacional ${ }^{4}$ lo cual sentó las bases para potenciar su desarrollo (Wang, 2010). Otro ejemplo es la Agencia de Viajes Internacional Chama Gudao de Tibet (西藏茶马古道 国 际旅行 社) fundada con ese nombre el año 1997. Su director general Liu Zhen, en una noticia de 2005, comentaba que desde el año 2000 los turistas chinos comenzaron a realizar el viaje por la Ruta y en 2004 recibieron a más de 600 turistas extranjeros y 200 turistas chinos, y más de 200 viajeros realizaron la Ruta entre Yunnan y Tibet en coche.

En definitiva, durante la segunda mitad de la década de 1990 se la denominó por primera vez de forma explícita como Ruta Antigua de Té y Caballos, es decir, se le añadió el calificativo de "antigua". A partir del año 2000 comenzó el turismo en ella y a partir de 2009 entró en una etapa de rápido desarrollo, especialmente entre Sichuan y Tibet y entre Yunnan y Tibet. De acuerdo a los datos disponibles es 
significativo cómo en un primer momento los turistas extranjeros superaban a los chinos en los viajes a Tibet. Sichuan, Yunnan y Tibet son tres regiones que ofrecen atractivos turísticos desde el comienzo del desarrollo del turismo en China, tanto para el turismo interno como para el internacional. La gran diversidad étnica de la población que reside en ellas, y que se diferencia del grupo de población mayoritario del país, denominado han, es uno de sus principales atractivos que al mismo tiempo es potenciado por el Estado central y local de diferentes niveles (Hillman, 2003; Schriver, 2017). La Ruta Antigua de Té y Caballos es una oferta turística de nuevo cuño que vincula mediante el diseño de un itinerario o recorrido basado en la historia a zonas, pueblos y ciudades que por sí mismas ya son objeto de atracción. Por lo tanto, este producto compite desde el primer momento de su creación con destinos turísticos ya consolidados como Kunming, Pu'er, Dali, Shaxi, Lijiang, etc. que se han desarrollado ajenos a la Ruta, aunque en la actualidad también tratan de sumarse a la nueva marca en la medida en que pueden aumentar, aún más si cabe, la llegada de turistas internos e internacionales (Liu, Zhang, y Wang, 2008), pues a una parte del turismo internacional también le llama la atención el realizar viajes en ruta o itinerarios culturales, y Tibet, que forma parte de esta ruta, todavía constituye un gran atractivo para el extranjero, así como se ha puesto de moda para el turismo chino interno (Yang, Lin y Han, 2010; Yeh y Coggins, 2014).

La Ruta, entre otros lugares, conecta a tres inscritos en el listado del Patrimonio Mundial de la UNESCO: el Conjunto Histórico del Palacio del Potala de Lhasa en Tibet desde 1994; la Ciudad Vieja de Lijiang en 1997 y el Area Protegida de los Tres Ríos Paralelos de Yunnan en 2003. La rama de la Ruta de Sichuan a Tibet atraviesa importantes ciudades como la capital de provincia Chengdu; o Ya'an, considerada el origen del cultivo del té en China en las cercanas montañas Mengding; Kanding (Dartsedo en tibetano) importante ciudad-mercado tibetana la provincia de Sichuan y Chando ya en Tibet, entre otras. Históricamente el mayor flujo comercial se llevaba a cabo entre Sichuan y Tibet (Booz, 2016; Tsomu, 2016).

\section{El perfil del turista chino y sus experiencias en ambas rutas}

La mayoría de los turistas chinos en ambas rutas es joven y con una buena salud y forma física. Su edad se encuentra en la franja de 18-35 años y prefiere el turismo profundo de carácter personalizado por motivos de ocio, aventura y deporte. Además de los grupos compuestos por jóvenes, también existe en la Ruta Antigua de Té y Caballos un segmento de personas de 30-50 años que proceden de las principales ciudades como Beijing, Shanghai y Guangzhou (Lai, 2015) que suelen viajar en grupo por motivos que incluyen la aventura, descubrimiento y conocimiento. Todos ellos pertenecen a la clase media y disponen de ingresos económicos altos o el apoyo económico estable de los padres, junto a un nivel educativo elevado. En el Camino, muchos son estudiantes o trabajadores en España o en otros países de Europa que conocen bastante la cultura e historia de diferentes países europeos y/o de España, además de los que trabajan y viven en las principales ciudades chinas y cuentan con un buen domino de inglés e incluso español y experiencia turística previa, es decir, ni el Camino ni la Ruta han sido su primer viaje turístico.

Otra característica común es su gran interés en obtener una experiencia que marca la diferencia y les distingue en comparación con el turismo urbano de masas. En el Camino de Santiago, la mayoría eligen y realizan el trayecto de los últimos 100 kilómetros desde Sarriá a Santiago de Compostela ${ }^{5}$ durante una semana a pie de forma independiente, algunos han hecho el recorrido en bicicleta e incluso a caballo. En el caso de la Ruta Antigua de Té y Caballos su preferencia es el trayecto entre Yunnan y Tibet durante 10 o 11 días en grupos pequeños con amigos y familiares contratando el servicio de agencias turísticas locales que les proporcionan guía, chófer y coche todoterreno con tracción en las cuatro ruedas para recorrer la geografía complicada de la Ruta a lo largo de 2.000 kilómetros. Los guías locales tienen la experiencia y los conocimientos profesionales de la Ruta.

En ambos casos el viaje comienza antes de emprenderlo obteniendo información del camino/ruta sobre la cultura, historia, folklore a la que acceden de diferentes formas, así como sobre aspectos más prácticos relacionados con la preparación del viaje. A nivel formal/institucional quien proporciona información sobre las rutas turísticas son las agencias de viaje. También las ferias dedicadas al turismo en China ofrecen información, por ejemplo, Turespaña en China ha impulsado las ofertas turísticas de Madrid, Barcelona, Sevilla y de otras ciudades de España, incluyendo la promoción del Camino de Santiago de Galicia. También se han organizado seminarios y mesas redondas para presentar y compartir experiencias turísticas de ambas rutas por parte de viajeros que las han realizado. 
La publicación de libros y guías por viajeros que narran sus experiencias en los mismos es otro medio importante de difusión. Con respecto al Camino de Santiago desde 2012 se han publicado en China varios libros (Xing, 2012; Wang, 2013). Igualmente ha ocurrido sobre la Ruta Antigua de Té y Caballos: el libro 嫁给318国道 (Se casa con la carretera nacional 318) redactado por el Departamento de Turismo de las Carreteras Chinas se publicó en 2005 y presenta a la carretera nacional 318 como la más larga y peligrosa y también la más atractiva de China. La revista Geografía Nacional China publicó en 2006 un número especial sobre la carretera nacional 318 que abordaba diferentes aspectos de la misma entre los que destaca su relación con la Ruta Antigua de Té y Caballos. A la carretera nacional 318 se la considera la Ruta Moderna de Té y Caballos.

Otra forma de acceder a la información sobre estas rutas/caminos son películas, documentales y series de televisión documentales y de ficción dedicadas a ellas o cuya trama se localiza en ellas El año 2004 se estrenó Delamu, película documental de Tian Zhuangzhuang. Rodada en la Ruta Antigua de Té y Caballos presenta la vida de los habitantes locales de la Ruta, especialmente de Yunnan, Sichuan y la Montaña Hengduan de Tibet (Lo, 2009). La película atrajo a muchos jóvenes que quedaron impactados por el argumento y fueron a visitarla convirtiéndose en los primeros turistas que realizaron este itinerario cultural. La serie documental de ocho capítulos también dirigida por Tian Zhuangzhuang, La Ruta Antigua de Té y Caballos (Chamagudao xilie: Delamu) se estrenó en la cadena nacional de televisión CCTV9 el año 2012. Otra serie documental de televisión de seis capítulos titulada Chamagodo (Asian Corridor in Heaven) sobre la historia, la cultura, el folklore y los recursos naturales de la Ruta Antigua de Té y Caballos fue realizada por KBS de Corea de Sur en el año 2007. En 2005 la Televisión Central de China emitió una teleserie dramática de 23 capítulos titulada La Ruta Antigua. Ambientada en 1942, presenta la historia de tres protagonistas de Yunnan durante la guerra sino-japonesa que ayudan a la caravana de mercaderes compuestas por personas de grupos étnicos distintos que mostraron su solidaridad para ayudar a transportar mercancías a la zona de guerra, enfatizando los aspectos patrióticos. Con respecto al Camino de Santiago la película The Way (2010), dirigida por Emilio Estévez, describe un viaje por el mismo. Un médico de Estados Unidos va a Francia para recoger los restos de su hijo Daniel fallecido en los Pirineos durante una tormenta cuando hacía el Camino de Santiago. El padre decide realizar el Camino de Santiago para comprender y experimentar lo que su hijo iba a hacer y finalmente encuentra el significado de su vida durante el recorrido. La historia conmovedora, los paisajes naturales y las viviendas y edificios antiguos que aparecen en la película han tenido una gran influencia sobre los viajeros chinos. Aunque esta película se exhibió en las salas de cine de China, la mayoría la vio en Internet y fue un estímulo para la toma de decisión de realizar el Camino de Santiago.

Existen portales de internet dedicados a los viajes como 马 蜂 窝 Mafengwo.cn, 一 起游 Yiqiyou (17U.com), 驴评网 Lvping.com, 穷游网 Qiongyouwang (qyer.com) que ofrecen información sobre los itinerarios, consejos para la preparación y experiencias personales de los turistas en ambas rutas. Los blogs de viajeros que comparten su experiencia turística son especialmente importantes como fuente de información para muchos aspectos prácticos, por ejemplo los bloggers Apito 麻 麻 del Camino de Santiago y 沉 墨 (Chenmo) de la Ruta Antigua de Té y Caballos. Los bloggers explican los trayectos, el equipamiento necesario, los medios de transportes, los puntos de interés a visitar, su experiencia diaria, los alojamientos, la gastronomía, apuntes sobre la historia y cultura peculiares que se encuentran, ofrecen consejos importantes para evitar los peligros y resolver problemas, por ejemplo, los números de teléfono de emergencias en caso de accidente y también muestran los fotos de su viaje a lo largo del camino.

En la actualidad todavía existe muy poca información completa y detallada sobre el Camino de Santiago ofrecida por canales oficiales en China (agencias de viaje). Por lo tanto, la mayoría de los turistas chinos accede a la información que necesita utilizando las posibilidades que ofrece internet: blogs de experiencias personales turísticas que explican detalladamente la información más útil para realizar el viaje. En el caso de la Ruta hay más opciones de acceso a la información, e incluso las agencias de viaje y turísticas offline organizan y ofrecen paquetes turísticos de la Ruta y proporcionan servicios de consulta e información.

Habitualmente para ambas rutas los turistas eligen la época de las vacaciones como la "semana dorada" en torno al 1 de octubre que es la Fiesta Nacional; o durante el Año Nuevo Chino entre enero y febrero; o para la Fiesta del Trabajo del 1 de mayo cuando también disponen de varios días de vacaciones que pueden utilizar para viajar. Los siete días de las vacaciones nacionales, más una parte de las anuales que les corresponden, facilita a los turistas realizar este tipo de viaje que requiere más tiempo. Además, debido a que el Camino se realiza viajando a pie, la mayoría prefiere hacerlo durante la temporada de otoño e invierno frente al verano que es más exigente físicamente. Los turistas de Corea del Sur, por ejemplo, se encuentran entre los que más viajan de noviembre a marzo y desde 2016 lideran el ranking 
de nacionalidades que hacen el Camino de Santiago en Enero (Coco, 2016). Los chinos muestran una preferencia semejante a la de los coreanos para realizar el Camino durante los meses de invierno. En el caso de la Ruta, en invierno es cuando hay más nieve lo cual da lugar a paisajes naturales de montañas nevadas y lagos helados que no se pueden visitar en verano, y es un atractivo añadido que les impulsa a realizar la Ruta en otoño/invierno. En el caso de los estudiantes que hacen el Camino, aprovechan sus vacaciones de verano e invierno para hacerlo.

Para preparar el viaje se informan por las experiencias de los bloggers y también por las agencias turísticas (Arlt y Aceves Leyva, 2011). En el Camino de Santiago los albergues son la principal forma de alojamiento, por lo que llevan sacos de dormir, almohadas y sábanas para crear un espacio que les resulte más cómodo y limpio. En la Ruta, en ocasiones, se alojan en templos o en caravasares para tener una experiencia especial. Y en ambos casos, cuando llegan al destino -Lhasa o Santiago de Compostela-, alquilan habitaciones en hoteles más caros. En la Ruta para evitar el problema de no aclimatarse y la falta de costumbre a la comida local picante de Sichuan, Yunnan y Tibet, aunque la prueban, también suelen llevar comida rápida para el viaje -galletas, fideos instantáneos, etc. Por el contrario, en el Camino de Santiago no tienen esa preocupación: son jóvenes que se adaptan a comidas diferentes y nuevas como la española y gallega que se ofrece en los bares y restaurantes a lo largo del viaje (Hotels.com, 2013).

Las actividades de los turistas chinos en el Camino y la Ruta son similares en muchos aspectos: se levantan muy temprano y empiezan el trayecto diario. Cuando se encuentran ante paisajes naturales y puntos de interés cultural y/o religioso paran y los visitan durante 30 minutos o una hora, para conocer de cerca su historia y la cultura asociada a los mismos (Long, 2014). En ambas rutas les gusta hacer fotografías en los paisajes llamativos y con las personas locales para evocar el recuerdo de haberlos visitado. Es habitual en ambos casos que compartan con sus amigos y familiares su experiencia diaria en las redes sociales mediante internet subiendo fotografías y textos en cuanto tienen conexión wifi.

Tienen mucho interés y sensibilidad sobre las peculiaridades de la religión, la cultura y la historia de los lugares por donde pasan (Lei, 2014) y consultan sobre los templos, iglesias, pueblos de las minorías étnicas, puntos turísticos de caravana y de producción de té, que visitan. Además, los turistas que hacen estos caminos/rutas están dispuestos y se muestran abiertos a conocer y participar en actividades culturales diferentes a lo largo del viaje. Por ejemplo, en el Camino saludan a los otros peregrinos, e imitan el ritual de poner piedras en los mojones que encuentran. Un atractivo especial son los sellos que ponen los lugares por donde se pasa, necesarios para conseguir el certificado que acredita haber realizado el Camino -la compostela. En la Ruta intentan saludar a los habitantes de las minorías étnicas en sus idiomas y al mismo tiempo aceptan el khata blanco tradicional que se les ofrece como bienvenida en las zonas tibetanas. Su interés se demuestra, por ejemplo, cuando después de los primeros días en que se quejan por la dificultad física que conlleva realizar ambas rutas, al final afirman que han disfrutado mucho la experiencia turística cultural que han vivido en ambas rutas.

Por otro lado, sobre las compras y el consumo, los turistas en las dos rutas consumen aquello que resulta básico a nivel cotidiano y al final de cada día, en el destino de la etapa, compran todo lo necesario para afrontar los problemas y las dificultades que hayan encontrado o que se prevé que afrontarán. También compran artículos propios de los lugares por donde pasan para guardar como recuerdo del viaje y para regalar a sus familiares y amigos. Por ejemplo, los ornamentos de las minorías étnicas y el té pu'er de la Ruta Antigua de Té y Caballos, y los ornamentos de conchas que simbolizan al Camino de Santiago. Es habitual que compren tarjetas postales con las imágenes de los paisajes locales representativos para enviarlas a sus familiares y amigos. Cada noche cuando llegan a los hoteles y albergues informan sobre su estado a los padres y familiares comentándoles que están fuera de peligro y no han tenido contratiempos. Los padres de los jóvenes se preocupan mucho por su seguridad, y por lo tanto, les informan para que estén tranquilos.

En el Camino los viajeros son más flexibles debido a la forma independiente del turismo. Cuando llegan al destino final de Santiago de Compostela, muchos también realizan viajes de un día a pueblos y ciudades cercanos, por ejemplo, A Coruña, Finisterre, etc. También se comunican con otros peregrinos a lo largo del viaje fuera de sus grupos, hablando y compartiendo sus experiencias del Camino e incluso hacen nuevos amigos. En cambio, en la Ruta, los viajeros mantienen sólo la relación y comunicación dentro de su pequeño grupo.

La motivación declarada de los turistas en ambos casos es el atractivo de la naturaleza (Liu, 2003), los paisajes de las montañas, lagos, campos, estepas, y los recursos culturales religiosos (Shen, 2005), del té, caravanas y minorías étnicas (Lei, 2014). En segundo lugar, a lo largo del viaje la mayoría de los viajeros tiene experiencias turísticas variadas de aventura, deporte (Luo, 2008), naturaleza, religión, y folklore. En tercer lugar, se muestran satisfechos de las infraestructuras turísticas (Zhang, 2011) como 
transportes, hoteles, albergues y restaurantes. En cuarto lugar, comparten sus experiencias exclusivas turísticas con los amigos y las familias y les recomiendan que realicen el viaje de ambas rutas. Eso da lugar a que cada vez más turistas chinos con un perfil similar eligen hacer estas rutas durante los últimos años. La única diferencia es que los jóvenes en el Camino prefieren la forma independiente (Lai, 2015) del turismo profundo (Yu, 2011) a pie con motivo de conseguir la experiencia turística con más contenido de aventura y los que hacen la Ruta y realizan el viaje en grupo, se inclinan más hacia la experiencia turística de ocio con más observación de la cultura (Reisinger, 1994).

Al fin y al cabo, para los turistas chinos de ambas rutas, el proceso del viaje duro (Zhang, 2001), que incluye observar la cultura, la religión y la historia junto a las dificultades que entraña el viaje, es lo que marca la diferencia y, en comparación al turismo urbano, lo que ellos hacen es un tipo de viaje sagrado espiritual que implica un esfuerzo personal. El viaje, a lo largo de la historia china, tiene asociaciones positivas, especialmente cuando se refiere a los de personas sabias o ejemplares que los emprendieron. La cultura china valora que la sabiduría y el conocimiento excepcional se pueden lograr a través de los viajes que lo buscan. Además, también valora positivamente la manera o el modo de alcanzar los ideales y el proceso para obtener resultados y logros, y en este sentido presta atención a la descripción de la experiencia del proceso del viaje (Zhang, 2001).

\section{Conclusión: Caminos en construcción y turistas independientes}

Ambas rutas/caminos de España y China, desde el punto de vista histórico, tienen su origen en la misma época. Desde el comienzo, aunque con diferente incidencia en distintos momentos de su historia, las dos rutas/caminos incluyen y conllevan importantes componentes económicos y religiosos. En el aspecto económico, la Ruta Antigua de Té y Caballos era una ruta comercial que dinamizaba la economía de las regiones que intercambiaban sus productos y el Camino de Santiago, con la gran movilidad de peregrinos que lo realizaron durante la Edad Media, y en la actualidad, afectó al desarrollo económico de todos los pueblos y ciudades que atravesaba con la creación de una considerable infraestructura de servicios y comercial. Con respecto al aspecto religioso, en el caso del Camino de Santiago es evidente porque era una ruta fundamentalmente de peregrinación religiosa y en la Ruta de Té y Caballos, a pesar de su carácter comercial, también la hacían peregrinos budistas en su camino a Lhasa y se difundieron ideas y creencias religiosas a lo largo de la misma, poniendo, además, en contacto a personas con diversas creencias.

El fenómeno del turismo, en ambos casos, comienza en los años noventa gracias a la difusión y recuperación por parte de los respectivos gobiernos, que ven en ellas motores de desarrollo local con posibilidades -turísticas- sin explotar: los recursos naturales y culturales -patrimonio material e intangible- son sus atractivos fundamentales. Ambos Estados han fomentado su desarrollo e intervenido en la planificación turística. Gradualmente se han convertido en itinerarios importantes del turismo cultural, uno ya consolidado, el Camino, y otro en plena construcción, la Ruta.

El turismo en China, tanto interno como internacional, conforme el nivel de vida del país y la clase media aumentan, es un servicio cada vez más demandado y la segmentación de la oferta ha crecido exponencialmente. Unos de los grupos más destacables en estos momentos son los jóvenes con ingresos considerables, nivel alto de estudios, abiertos y con expectativas de tener experiencias novedosas que los alejen y distancien del resto de la población. Son ellos quienes buscan y se informan por su cuenta y están interesados en viajes "diferentes", "lentos"-slow travels-, independientes y personalizados frente al viaje "con prisas", o de compras, que sin dejar de existir, ya no son la única opción. Croce (2016) elabora una tipología del turismo chino cuyo destino es Europa distinguiendo a los tradicionales (70\%), jóvenes cultos (wenyi) (10\%), centrados en experiencias (10\%), expertos/entendidos (7\%) y hedonistas (3\%). Por su parte Xu y Wang (2014: 9) en la tipología sobre nuevas tendencias emergentes de modos de viaje al extranjero incluyen a "independent tours, island tours, in-depth experiential tours, and high-end outbound tours and cruise tours".

El caso aquí presentado es un ejemplo de turismo chino interno e internacional en itinerarios culturales que llevan a cabo personas con características, inquietudes y expectativas similares. Todavía su volumen es pequeño en comparación con el turismo masificado que ya existe en China, pero es una tendencia en aumento que obliga a cambiar la imagen y percepción estereotipada sobre el turismo chino.

Las redes sociales e internet son la clave que facilita tanto el acceso directo a la información como la posibilidad de organización independiente de los viajes (compra de billetes de transporte, reservas de alojamiento, etc.) a la que cada vez recurren más los turistas chinos. Conforme se abren nuevas 
posibilidades, son inmediatamente aprovechadas por ellos. Las rutas/caminos aquí presentadas implican un esfuerzo físico especial, junto a un plus de aventura, improvisación y riesgo, hasta cierto punto novedoso en la sociedad china, pero al que una parte de la población, sobre todo joven y con un nivel educativo alto, le resulta atractiva como experiencia. La cultura -material e inmaterial- mientras tanto, se consume a lo largo del camino.

\section{Bibliografía}

Agís Villaverde, Marcelino

2008. "Antropología de la Peregrinación: ¿Quiénes son los peregrinos?”. XI Encuentro de Santuarios de

España. Santiago de Compostela, 23-25 de noviembre. Conferencia Episcopal Española.

Andrade Suárez, María J., Leira Landeira, Grial y Caramés Valo, Rosa

2010. "El turismo como factor estratégico de desarrollo rural: el Camino de Santiago Francés". ROTUR.

Revista de Ocio y Turismo, 3 (1): 49-82.

Andreu, Rosario, Claver, Enrique y Quer, Diego

2013. "Chinese outbound tourism: New challenges for European tourism". Enlightening Tourism. A Pathmaking Journal, 3 (1): 44-58.

Arlt, Wolfgang G. y Aceves Leyva, Alma B.

2011. "El auge del turismo emisor de China: ¿qué buscan y cómo se comportarán 100 millones de nuevos turistas?”. Anuario Asia Pacifico 2010, edición 2011 (pp. 327-334). Barcelona: Casa Asia, Fundación CIDOB.

Booz, Patrick

2016. “'To control Tibet, first pacify Kham': Trade routes and 'official routes' (Guandao) in Easternmost Kham". Cross-Currents: East Asian History and Culture Review E-Journal, 19: 27-47. <http://cross-currents.berkeley.edu/e-journal/issue-19>.

Bovair, Elizabeth A.

2008. "Journeys to Shangri-La: The neo-Orientalism of Tibetan culture". En Cochrane, Janet (Ed.), Asian tourism: Growth and change (pp. 335-343). Amsterdam: Elsevier.

Calabuig Tomás, Jordi y Ministral Masgrau, Marta

1998. Manual de Geografía turística de España. Madrid: Editorial Síntesis.

Cànoves Valiente, Gemma y Blanco Romero, Asunción

2011. "Turismo religioso en España: ¿La gallina de los huevos de oro? Una vieja tradición, versus un turismo emergente". Cuadernos de Turismo, 27: 115-131.

Capel, Horacio

2005. "Las rutas culturales como Patrimonio de la Humanidad. El caso de las fortificaciones americanas del Pacífico". Biblio 3W. Revista Bibliográfica de Geografía y Ciencias Sociales [en línea]. 10 (562). $<$ http://www.ub.es/geocrit/b3w-562.htm>

CESTUR-SECTUR

2002. Estudio estratégico de viabilidad para el segmento de turismo cultural en México. [En línea]. México: Secretaría de Turismo y Centro de Estudios Superiores de Turismo. <http://cestur.sectur. gob.mx/pdf/estudioseinvestigacion/segmentosyproductos/productos/Estudiodeturismocultural.pdf> (consultado el 20 de mayo de 2018).

Chen, Baoya 陈 保 亚

2004. 论茶马古道的起源 (Lun chama gudao de qiyuan, Acerca del origen de la Ruta Antigua de Té y Caballos). 思 想 战 线 (Sixiang zhanxian), 30 (4): 44-50.

Chen, Baoya 陈 保 亚

2005 , 茶马古道: 横跨世界屋脊的文化传播纽带--纪念茶马古道首次徒步考察和 命名 15 周年 (Chama gudao: hengkua shijie wuji de wenhua chuanbo niudai- jinian chama gudao shouci tubu kaocha he mingming 15 zhounian, La Ruta Antigua de Té y Caballos: vínculo cultural mundial - 15 años de recuerdo del primer trabajo del campo a pie y denominación del nombre de la ruta). 科学 中国 人 (Kexue Zhongguoren) 12: 16-19.

Coco, Abraham

2016. "Los coreanos peregrinan en enero". ABC Galicia, 2 de febrero de 2016. <https://www.google.es/ amp/www.abc.es/espana/galicia/abci-camino-santiago-coreanos-peregrinan-enero-201602021130_noticia_amp.html> (consultado el 20 de mayo de 2018). 
Craik, Jennifer

1997. "The culture of tourism". En Rojek, Chris y Urry, John (Eds.), Touring cultures: Transformations of travel and theory (pp. 113-136). Londres: Routledge.

Croce, Valeria

2016. Tourism in focus. The Chinese outbound travel market. Virtual Tourism Observatory. Directorate-General for Internal Market, Industry, Entrepreneurship and SMEs. European Commission. <www. ec.europa.eu/growth/tools-databases/vto> (consultado el 20 de mayo de 2018).

Cubeiro, Dídac

2015. "La ruta del té. (Antigua ruta comercial de China a Tibet)". Historia y vida, 569: 56-61.

Estella, Sara

2017. "El Camino seduce al viajero solitario". La Voz de Galicia, 27 de agosto. <https://www.lavozdegalicia. es/noticia/mercados/2017/08/27/camino-seduce-viajero-solitario/0003_201708SM27P12991.htm> (consultado el 20 de mayo de 2018).

Fernández-Baca, Román

2014. "50 años de la Carta de Venecia: Del monumento histórico y su conservación al desarrollo sostenible". En López Morales, Francisco J. y Vidargas, Francisco (Eds.), Los nuevos paradigmas de la conservación del patrimonio cultural. 50 años de la Carta de Venecia (pp. 42-49). México: CONCULTA-INAH.

Fernández Salinas, Víctor

2013. "De dónde y hacia dónde. Perspectivas y premisas para el entendimiento de los itinerarios culturales". Biblio 3W. Revista Bibliográfica de Geografía y Ciencias Sociales [en línea]. 28 (1028). $<$ http://www.ub.es/geocrit/b3w-1028.htm>

Gómez Prieto, Julia y González-Quijano, Covadonga

1991. Rutas e itinerarios turísticos en España. Madrid: Editorial Síntesis.

Hernández, Emilio

2018. "El turismo chino en España: oportunidad y nuevas tendencias". ARI 48/2018. Madrid: Real Instituto Elcano.

Hernández Ramírez, Javier

2011. "Los caminos del Patrimonio. Rutas turísticas e itinerarios culturales". Pasos: Revista de Turismo y Patrimonio Cultural, 9 (2): 225-236

Hillman, Ben

2003. "Paradise under construction: Minorities, myths and modernity in Northwest Yunnan". Asian Ethnicity, 4 (2): 175-188.

Hotels.com

2013. "Chinese International Travel Monitor 2013”. Jing Daily, 15 de octubre de 2013.

$<$ https://jingdaily.com/chinese-international-travel-monitor-2013/> (consultado el 20 de mayo de 2018). ICOMOS

2008. Carta de Itinerarios Culturales. Quebec: Comité Científico Internacional de Itinerarios Culturales (CIIC) del ICOMOS. 6a Asamblea General del ICOMOS, 4 de octubre de 2008. <https://www.icomos. org/images/DOCUMENTS/Charters/culturalroutes_sp.pdf> (consultado el 20 de mayo de 2018).

Kolås, Åshild

2004. "Tourism and the making of place in Shangri-La". Tourism Geographies, 6 (3): 262-278.

Lai, Shengwei 赖 声 伟

2015. 中国自助游发展研究 (Zhongguo zizhuyou fazhan yanjiu, Investigación sobre el desarrollo del turismo de forma independiente de China). 中国商论 (Zhongguo shanglun, Century Journals Project - Economy \& Management (Series J) 24: 136-138.

Laso, Gregorio, y Justo, Pierre

2009. "La imagen de España en China". Boletín Económico del ICE, 2972: 95-110.

Lei, Junxia 雷 俊 霞

2014. 中国民俗旅游发展趋势研究 (Zhongguo minsu lüyou fazhan qushi yanjiu, Análisis sobre la tendencia del desarrollo del turismo chino de folklore). 旅游纵览月刊 (Lüyou zonglan yuekan) 6.

Litzinger, Ralph

2014. "Afterword. The afterlives of Shangrila". En Yeh, Emily T. y Coggins, Chris (Eds.), Mapping Shangrila. Contested Landscapes in the Sino-Tibetan Borderlands (pp. 279-286). Seattle: University of Washington Press.

Liu, Deqian 刘 德 谦

2003. 中国生态旅游的面临选 择 (Zhongguo shengtai lüyou de mianlin xuanze, Elección del turismo chino ecológico). 旅游学 刊 (Lüyou Xuekan), 18 (2), 63-68. 
Liu, Jianfeng, Zhang, Jie y Wang, Guiyu, 刘 建 峰, 张 洁, 王 桂 玉

2008. 基于 “点——轴系统“理论的云南茶马古道旅游发展战略研究 (Jiyu dian-zhou xitong lilun de Yunnan chama gudao lüyou fazhan zhanlue yanjiu, Investigación de la estrategia y desarrollo del turismo de la Ruta Antigua de Té y Caballos de Yunnan en base sobre la teoría del sistema de punto y eje). 桂 林旅游高等专科学校学报 (Guilin Lüyou Gaodeng Zhuanke Xuexiao Xuebao), 19 (1): 81-86.

Llamas, Rosa y Belk, Russell

2011. "Shangri-La: Messing with a myth". Journal of Macromarketing, 31 (3): 257-275.

Lo, Kwai-Cheung

2009. "Two moments of ethnic representation in Tian Zhuangzhuang's minority films". Journal of Chinese Cinemas, 3 (3): 231-247.

Lojo, Aureli

2016. "Turismo chino en España: un análisis del producto turístico, atracciones e itinerarios ofrecidos por agencias de viajes chinas". Cuadernos de Turismo, 37: 511-517.

Long, Jiangzhi 龙 江 智

2014. 中国旅游消费行为模式研究 (Zhongguo lüyou xiangfei xingwei moshi yanjiu, Estudio del modo del comportamiento del consumo del turismo chino). 旅游教育出版社 (Lüyou jiaoyu chubanshe).

López-Guzmán, Tomás J., Lara de Vicente, Fernando J., y Merinero-Rodríguez, Rafael

2006. "Las rutas turísticas como motor de desarrollo económico local. La ruta del Tempranillo". Estudios Turísticos, 167: 131-145.

Lui, Vincent, Kuo, Youchi, Fung, Justin, Jap, Waldemar y Hsu, Hubert

2011. Taking off. Travel and tourism in China and beyond. Boston: The Boston Consulting Group Report. $<$ https://www.bcg.com/documents/file74525.pdf> (consultado el 20 de mayo de 2018). Luo, Qing 罗清

2008.中国生态旅游发展前景分析 (Zhongguo shengtai lüyou fazhan qianjing fenxi, Análisis del desarrollo del turismo chino ecológico). 消 费 导 刊 (Xiaofei Daokan) 16: 44-45.

Luo, Shiwei 罗世 伟

2003. 茶马古道历史线路与旅游开发现实意义(Chama gudao lishi luxian yu lüyou kaifa xianshi yiyi, Trayecto histórico de la Ruta Antigua de Té y Caballos y su desarrollo turístico [J]. 重 庆 师范大学学报 (自然科学版) (Chongqing shifan daxue xuebao (ziran kexue ban)), 20 (3): 54-57.

Morère Molinero, Nuria

2012. "Sobre los itinerarios culturales del Icomos y las rutas temáticas turístico-culturales. Una reflexión sobre su integración en el turismo”. Revista de Análisis Turístico, 13: 57-68.

$\mathrm{Mu}$, Jihong, 木 雯 弘.

2001. 茶马古道考察纪 事 (Chama gudao kaocha jishi, Trabajo del Campo en la Ruta Antigua de Té y Caballos). Kunming: 云 南 教育出版 社. (Yunnan Jiaoyu Chubanshe)

Ning, Shimin 宁 士 敏.

2000. 影响中国旅游消费的经济和社会因素分析 (Yingxiang zhongguo lüyou xiaofei de jingji he shehui yinsu fenxi, Análisis de los factores que influyen en el consumo del turismo chino). 游学 刊 (Lüyou xuekan), 15 (3): 75-76.

Noya, Javier et al.

2007. La imagen de España en China. Madrid: Real Instituto Elcano de Estudios Internacionales y Estratégicos.

Prnoticias

2018. “Todas las noticias del turismo chino en España durante 2017". Prnoticias, 22 de enero. <https:// prnoticias.com/marketing/prmarketing/20166965-todas-las-cifras-del-turismo-chino-en-espana-durante-2017> (consultado el 20 de mayo de 2018).

Reisinger, Yvette

1994. "Tourist-host contact as a part of cultural tourism". World Leisure and Recreation, 36 (2): 24-28.

Richards, Greg

2001. "El desarrollo del turismo cultural en Europa". Estudios Turísticos, 150: 3-14.

Sánchez, Jorge

2005. El Camino del peregrino a Santiago. Madrid: Manakel.

Schriver, Silvia

2017. Cultural heritage preservation in regional China: Tourism, culture and the Shaxi model. Tesis doctoral. Perth: The University of Western Australia. 
Shen, Zuxiang 沈 祖 祥.

2005. 中国宗教旅游 (Zhongguo zongjiao lüyou, Turismo religioso chino). 福建 人民出版社. Fuzhou: (Fujian renmin chubanshe).

Sigley, Gary

2010. "Cultural heritage tourism and the Ancient Tea Horse Road of Southwest China". International Journal of China Studies, 1 (2): 531-544.

2015. "Tea and China's rise: Tea, nationalism and culture in the 21st century". International Communication of Chinese Culture, 2 (3): 319-341.

Tomás Gaimúndiz, Daniel

2011. El turismo chino en España: La adaptación de destinos turísticos españoles al mercado turístico más grande del mundo. Tesis Fin de Máster. Sevilla: Universidad de Sevilla.

Torres Bernier, Enrique

2006. "Rutas culturales. Recurso, producto y destino turístico". Revista PH, Boletín del Instituto Andaluz del Patrimonio Histórico, 60: 84-97.

Tsomu, Yudru

2016. "Guozhuang trading houses and Tibetan middlemen in Dartsedo, the 'Shanghai of Tibet". Cross-Currents: East Asian History and Culture Review E-Journal, 19. <http://cross-currents.berkeley. edu/e-journal/issue-19>

UNESCO,

2002. The Silk Roads Project: Integral study of the Silk Roads: Roads of Dialogue, 1988-1997. CLT/ CPD/DIA/2008/PI/68. <http://unesdoc.unesco.org/images/0015/001591/159189E.pdf> (consultado el 20 de mayo de 2018).

Vivential Value

2016. Monitor de Mercados Online - Edición China 2016, en "Barcelona es el destino líder en reputación online para los turistas chinos", CETT-UB Noticias, 19 de septiembre. <https://www.hosteltur.com/ comunidad/nota/004495_barcelona-es-el-destino-lider-en-reputacion-online-para-los-turistas-chinos. html> (consultado el 20 de mayo de 2018).

Wang, Sainan 王 赛 男

2013. 圣 地亚哥朝 圣之 路 (Shengdiyage chaosheng zhi lü, Camino de Santiago). Wuhan: 武 汉 大学出版 社 (Wuhan daxue chubanshe).

Wang, Zhongqiang 王 忠 強

2010. 千年茶马古道 (Qiannian chama gudao, Ruta Antigua de Té y Caballos milenaria). Jilin: 吉 林 文 史出版 社 (Jilin wenshi chubanshe).

World Tourism Organization (UNWTO)

2017 Penetrating the Chinese outbound tourism market. Successful practices and solutions. Madrid: UNWTO.

World Tourism Organization (UNWTO)

2018. Tourism and culture synergies. Madrid: UNWTO. DOI: https://doi.org/10.18111/9789284418978

Xing, Xiaosheng 刑 啸声

2012. 圣雅各之路朝 “胜” 记 (Shenyage zhi lu chao “sheng” ji, Notas de viaje del Camino de Santiago). 桂 林 (Guilin): 广西师范大学出版 社 (Guangxi shifan daxue chubanshe).

$\mathrm{Xu}$, Fan y Wang, Suosheng

2014. "China's outbound tourism. Emerging trends and features in the second decade of the 21st century". Perspectives in Asian Leisure and Tourism, 2 (1): Article 2.

Yang, Chih-Hai, Lin, Hui-Lin y Han, Chia-Chun

2010. "Analysis of international tourist arrivals in China: The role of World Heritage Sites". Tourism Management, 31(6), 827-837.

Yeh, Emily T. y Coggins, Chris, eds.

2014. Mapping Shangrila. Contested landscapes in the Sino-Tibetan borderlands. Seattle: University of Washington Press.

Yu, Yang 于 洋

2011. 浅析中国乡村旅游发展 (Qianxi Zhongguo xiangcun lüyou fazhan, Análisis breve sobre el desarrollo del turismo chino rural). 管理 学 家 (Guanli xuejia), 9.

Zhang, Guohong 张 国 洪

2001. 中国文化旅游:理论, 战略, 实践 (Zhongguo wenhua lüyou: lilun, zhanlue, shijian, Turismo cultural de China: teoría, estrategia, práctica) 南开大学出版 社 (Nankai Daxue Chubanshe) 
Zhang, Jinghong

2014. Puer Tea: Ancient caravans and urban chic. Seattle: University of Washington Pres.

Zhang, Lijun 张 丽 君

2011. 自助旅游现状及其发展分析 (Zizhu lüyou xianzhuang ji qi fazhan fenxi, Situación actual del turismo de la forma independiente y análisis sobre su desarrollo). 中国商论 (Zhongguo Shanglun) 5: 173-174.

Zhang, Min y Ma, Shouchun 张 敏, 马 守 春

2006. 西藏昌都地区旅游业的现状及发展对策 (Xizang Changdu diqu lüyouye de xianzhuang ji fazhan duice, Situación actual y estrategias de desarrollo de la industria turística de la zona Changdu de Tíbet). 西部林业科 学 (Xibu Linye Kexue), 35 (3): 122-127.

Zhang, Ruiguang, 张广瑞.

2002. 中国旅游发展 (Zhongguo lüyou fazhan, Desarrollo del turismo chino). Beijing: 社会科学 文献出版 社 (Shehui kexue wenxi chubanshe, Editorial de Documentación de Ciencias Sociales).

Zhang, Yuan

2015. La peregrinación a Santiago de Compostela: una aproximación global y propuesta de una agencia de turismo en China sobre el Camino de Santiago. Trabajo Fin de Master. Valencia: Universitat Politècnica de València.

\section{Agradecimientos}

Esta investigación forma parte del proyecto I + D del Ministerio de Economía y Competitividad (FFI2015-70513-P MINECO/FEDER, UE) “Asia Oriental: paradigmas emergentes, política(s), dinámicas socioculturales y sus consecuencias", así como del Grupo de Investigación Consolidado (GRC) InterAsia y el nuevo sistema internacional: Sociedad, política y cultura (2017 SGR 1284) de la Generalitat de Catalunya.

\section{Notas}

1 Cada siglo cuenta con catorce años jacobeos, es decir, cuando coincide la fiesta de Santiago (25 de Julio) en domingo, que comportan la indulgencia plenaria o completa con la remisión de la pena temporal de todos los pecados cometidos por el peregrino. Cuando no es año jubilar, las indulgencias son menores.

2 Xunta de Galicia, Asociaciones de amigos del Camino, El Camino de Santiago. http://www.caminodesantiago.gal/es/ descubre/asociaciones-del-camino/asociaciones-de-amigos-del-camino (consultado del 20 de mayo de 2018).

3 El gobierno chino siempre presta atención a las conexiones e infraestructura de transporte en primer lugar con el fin de facilitar la llegada del mayor número de personas al destino turístico para su desarrollo.

4 El gobierno chino clasifica los lugares turísticos desde el nivel A al AAAAA en base a su calidad para recomendarlos y ofrecer garantías a los turistas. La categoría Premium es la que cuenta con cinco A.

5 Según los datos de 2017, de las 180.737 personas que realizaron el Camino Francés (60,04\% del total), 79.717 (el 26,48\% del total) hicieron el trayecto desde Sarria a Santiago de Compostela. 